J. Genet., Vol. 64, No. 1, July 1985, pp. 41-58. (C) Printed in India.

\title{
Evolution of sex ratios in social hymenoptera: kin selection, local mate competition, polyandry and kin recognition
}

\author{
N V JOSHI and RAGHAVENDRA GADAGKAR* \\ Centre for Theoretical Studies and *Centre for Ecological Sciences, \\ Indian Institute of Science, Bangalore 560012, India.
}

MS received 24 May 1985

\begin{abstract}
A model is constructed to study the effects of local mate competition and multiple mating on the optimum allocation of resources between the male and female reproductive brood in social hymenopteran colonies from the 'points of view' of the queen (parental manipulation theory) as well as the workers (kin selection theory). Competition between pairs of alleles specifying different sex investment ratios is investigated in a game theoretic frame work. All other things being equal, local mate competition shifts the sex allocation ratio in favour of females both under queen and worker control. While multiple mating has no effect on the queen's optimum investment ratio, it leads to a relatively male biased investment ratio under worker control. Under queen control a true Evolutionarily Stable Strategy (ESS) does not exist but the 'best' strategy is merely immune from extinction. A true Ess exists under worker control in colonies with singly mated queens but there is an asymmetry between the dominant and recessive alleles so that for some values of sex ratio a recessive allele goes to fixation but a dominant allele with the same properties fails to do so. Under multiple mating, again, a true ESS does not exist but a frequency dependent region emerges. The best strategy here is one that is guaranteed fixation against any competing allele with a lower relative frequency. Our results emphasize the need to determine levels of local mate competition and multiple mating before drawing any conclusions regarding the outcome of queen-worker conflict in social hymenoptera. Multiple mating followed by sperm mixing, both of which are known to occur in social hymenoptera, lower average genetic relatedness between workers and their reproductive sisters. This not only shifts the optimum sex ratio from the workers' 'point of view' in favour of males but also poses problems for the kin selection theory. We show that kin recognition resulting in the ability to invest in full but not in half sisters reverts the sex ratio back to that in the case of single mating and thus completely overcomes the hurdles for the operation of kin selection.
\end{abstract}

Keywords. Sex ratios; kin selection; local mate competition; polyandry; kin recognition; social hymenoptera.

\section{Introduction}

Fisher (1930) showed that in outbreeding populations natural selection would favour equal parental investment in the offspring of each sex. If the population is inbreeding however, competition for females is predominantly between brothers (local mate competition) so that natural selection favours a female biased sex ratio (Hamilton 1967). In the extreme case, when there is complete sibmating, a parent 'should' produce just enough sons necessary to inseminate all the daughters. These predictions of Fisher and Hamilton have been repeatedly verified, both theoretically and empirically (Charnov 1982; Metcalf 1980; Owen 1983; Werren 1980, 1983). Indeed the theory of sex allocation has in recent years become a cornerstone of evolutionary biology, a status achieved primarily because of the success with which precise quantitative and empirically testable predictions have been made (Charnov 1982). 
The theory of sex allocation has assumed importance for yet another reason. Predictions concerning sex allocation appear to be powerful in choosing between competing theories purporting to explain the evolution of sociality in insects (for a recent review see Gadagkar 1985a). A hidden assumption in Fisher's argument of equal allocation between the two sexes is that a parent is equally related to his or her sons and daughters. In haplodiploid social insects such as wasps, bees and ants, sterile female workers often feed and care for their siblings instead of producing their own offspring. Haplodiploidy, a genetic system where males arise from haploid unfertilized eggs and females from fertilized, diploid eggs, creates asymmetries in genetic relatedness. Females are related to their sisters by $3 / 4$ and to their brothers by $1 / 4$. This asymmetry is in fact an important factor in favour of Hamilton's $(1964 \mathrm{a}, \mathrm{b})$ theory of the evolution of social behaviour because it genetically predisposes a hymenopteran worker towards the evolution of altruistic behaviour. Trivers and Hare (1976) argued, however, that a worker gains nothing in fitness if she invested equally in brothers and sisters because her average relatedness to her siblings is $1 / 2$, the same as her average relatedness to her own offspring. Sterility in workers would be selected if they can capitalise on the asymmetries in genetic relatedness by investing in their sisters and brothers in the ratio $3: 1$. In other words, when workers invest in siblings who are related to them in the ratio $3: 1$, natural selection would favour a ratio of allocation paralleling the ratio of relatedness. The queen who is fertile and who produces the sons and daughters would on the contrary favour an equal investment in brood of the two sexes in her colony. In this context there would be a conflict of interests between the queen and the workers in the optimum ratio of allocation of resources between brood of the two sexes.

The theory of kin selection argues that workers are sterile and act altruistically towards their sibs because this is the strategy that maximises their inclusive fitness. Inclusive fitness of an individual may be defined as its total contribution to the gene pool of the next generation obtained both by the production of offspring and by aiding genetic relatives. The theory of parental manipulation (Alexander 1974) on the other hand suggests that workers are sterile because they are manipulated into this state by their parents. The two theories make mutually opposing predictions regarding the expected allocation between the two sexes in the reproductive brood in social hymenopteran colonies. If kin selection is responsible for worker sterility and altruism, then the workers should win in the conflict over the investment ratio and the resultant sex-investment ratio observed should be $3: 1$. If worker sterility and altruism are a consequence of parental manipulation instead, queens should be successful in manipulating the workers into investing equally in reproductives of the two sexes and a 1:1 ratio of investment is expected. Trivers and Hare (1976) weighed male and female reproductives in a number of monogynous ant colonies and showed that the observed ratios of investment were significantly closer to 3:1 than 1:1 and concluded that their data are uniquely explained by kin selection theory.

The predictions and conclusions of Trivers and Hare (1976) depend on the assumption that the social insects under consideration are outbreeding and that the queens mate only once. Under inbreeding or local mate competition (LMC), the queens too would prefer a female biased investment ratio (Hamilton 1967) and the 3:1 ratio seen by Trivers and Hare may have nothing to do with workers realising their optimum investment ratio as opposed to the queens' optimum value (Alexander and Sherman 1977). Similarly, if the queens mate with more than one male then the relatedness between the workers and the reproductive sisters they rear will no longer be $3 / 4$ but distributed anywhere between $3 / 4$ (full sisters) and $1 / 4$ (half sisters). Under these 
conditions the predictions used by Trivers and Hare (1976) are no longer valid (Alexander and Sherman 1977). Both LMC and multiple mating by queens are known to occur in social hymenoptera although their intensities might vary widely (Alexander and Sherman 1977; Page and Metcalf 1982; Crozier 1980). While multiple mating has long been recognised to be common in hymenoptera (see Wilson 1971) it has often been assumed that sperms from different males clump in the spermatheca of the females leading to use of sperm from a single male for extended periods of time (see Orlove 1975, for example; see Crozier and Brückner 1981, and Starr 1984, for detailed discussions of other available data). However, in the only case where careful investigation has gone into this question it is clear that sperms do not clump (Page and Metcalf 1982).

When multiple matıng results in a lowered average genetic relatedness between workers and reproductives in a colony this not only alters the expected sex-investment ratio from the workers' 'point of view' but may also be considered as a factor against kin selection (Hamilton 1964b; Wilson 1971). It may be argued however that 'workers can circumvent the problem of multiple insemination' by kin recognition leading to investment in full sisters but not in half-sisters (Page and Metcalf 1982).

In recent years a number of theoretical investigations relating to the evolution of sex ratios in social hymenoptera have been undertaken. Stimulated in part by the work of Trivers and Hare (1976) who predicted a 3:1 investment ratio under worker control and a 1:1 ratio under queen control, several authors have confirmed these predictions by rigorous methods (Oster et al 1977; MacNair 1978; Craig 1980a; Uyenoyama and Bengtsson 1981; Charnov 1978a). Other factors such as LMC and worker-queen conflict have also occasionally been considered (Taylor and Bulmer 1980; Oster et al 1977; Bulmer 1981; Benford 1978). Our intention here is to simultaneously consider the effects of LMC, polyandry and kin recognition and generate predictions regarding optimum sex allocation ratios in the frame work of the kin selection and the parental manipulation theories. In this paper we are only working within the context of the Trivers and Hare (1976) and Alexander and Sherman (1977) arguments in our efforts to recalculate what in fact should have been the theoretical predictions of the former authors, had they taken the points made by the latter into consideration. It should be mentioned that the importance of female biased sex ratios in the evolution of eusociality is not universally agreed upon. Craig's $(1979,1980 \mathrm{~b})$ models for instance suggest that female biased sex ratios are not likely to have been very useful for the origin of eusocial behaviour. In the maintenance of already existing eusocial behaviour, Charnov (1978b) points out that selection for egg laying by workers is very strong even if they are rearing a "seemingly advantageous" combination of brothers and sisters.

\section{The model}

We consider an infinite population of social insect colonies. Each colony is initiated by a single inseminated female (queen), whose all-female first brood consists entirely of workers. The subsequent brood consisting of both male and female reproductives is fed and cared for by the workers. The queen dies after the emergence of reproductives. From each nest a fraction $d$ of both male and female reproductives disperses to join a mating aggregate where random mating takes place. On the other hand, sibmating takes place within the fraction $(1-d)$ remaining at each nest. After mating the males die and each inseminated female initiates a new nest. Thus $d$ parametrises local mate 
competition, with $d=1$ corresponding to complete outbreeding, and $d=0$ complete inbreeding. We have of course modelled LMC in this fashion for convenience. LMC could occur even in the absence of sibmating if, for instance, the males from one nest all attempt to mate with the females of a neighbouring, though unrelated nest.

The sex ratio trait is modelled by a one-locus-two allele system. The allele $A$ being dominant, individuals with genotype $A A$ and $A B$ produce a fraction $r_{A}$ of males among their reproductive progeny, while those of genotype $B B$ produce a fraction $r_{B}$ of males. For simplicity we have assumed that investment ratio is directly translated into sex ratio.

\subsection{Queen control of the investment ratio}

Even if the optimum investment ratio is different for the queen and the workers, the queen could in principle manipulate the workers into feeding her reproductive offspring in the ratio optimum for her. This is modelled by adjusting the sex ratio of the reproductive offspring according to the genotype of the queen.

If queens mate only once, there will be six types of inseminated females, $A A . A, A A . B$, $A B . A, A B . B, B B . A$ and $B B . B$ (where the first two letters refer to the queen's genotype and the third to the genotype of the male she has mated with i.e., of the sperm stored in her spermatheca). Each inseminated female contributes genes to the next generation by three pathways: (1) through the sons which join the mating aggregate, (2) the daughters which join the mating aggregate, (3) the sibmated daughters. The quantitative details about the contributions from each of the six types of inseminated females are described in table A1 (appendix).

Thus knowing the frequencies $P_{A A . A}(n), P_{A A . B}(n) \ldots, P_{B B . B}(n)$ of each of the classes in the $n$th generation, one can obtain the frequencies $P_{\text {AA.A }}$ $(n+1), \ldots$ etc. in the next generation. Since all the frequencies add up to unity, the dynamics is described by a system of five coupled nonlinear difference equations (see appendix).

To study the competition between two alternative sex ratio strategies $r_{A}$ and $r_{B}$, we consider a population purely of type $A A . A$, into which a small proportion of inseminated females containing the $B$ gene is introduced. For this situation, the above system of five nonlinear coupled equations can be approximated by a system of five linear difference equations. As shown in the appendix, the elements of the relevant transformation matrix $G^{\prime}$ are functions of $r_{A}, r_{B}$ and $d$ alone.

If the dominant eigenvalue of the matrix $G^{\prime}$ is greater than unity the proportion of $B$ increases with time and we say that $A$ is invadable by $B$. Conversely, if the eigenvalue is less than unity, $A$ is uninvadable by $B$. Similarly, we can investigate whether a pure population of $B$ is invadable by $A$.

If pure $A$ is uninvadable by $B$ while $B$ is invadable by $A$ then $A$ would be selected for and would go to fixation. If both pure $A$ and pure $B$ are uninvadable, frequency dependent selection is implied (whichever establishes itself first wins). Finally if both pure $A$ and pure $B$ are invadable, co-existence of the two alleles is indicated.

For different values of $r_{A}$ (the proportion of males specified by the dominant allele), $r_{B}$ (proportion of males specified by the recessive allele) and $d$, the parameter characterizing local mate competition, we have investigated the dynamics of the system to determine which of the above conditions prevail viz. one of the two alleles going to fixation, the two alleles coexisting, or frequency dependent selection. 


\subsection{Worker control of investment ratios}

Trivers and Hare (1976) assumed that "the offspring is capable of acting counter to its parents' best interests" and thus workers should be able to feed the reproductive brood in the ratio that optimises their inclusive fitness. Once again making the simplifying assumption that investment ratios are directly translated into sex ratios, this is modelled simply by adjusting the sex ratio of the reproductive brood in accordance with the genotype of the workers. For instance queens of the type $A B . B$ will produce workers of the type $A B$ and $B B$ in equal proportions. Although some investigators have considered the possibility of workers of one genotype behaviourally dominating over workers of other genotypes (Charnov 1978; Craig 1980; Pamilo 1982; Bulmer 1983) we agree with Bulmer (1983) that additivity seems biologically more likely. Thus in a colony with $A B$ and $B B$ workers in equal numbers, the proportion of males in the reproductive brood is taken to be $\left(r_{A}+r_{B}\right) / 2$. Thus $\left(r_{A}+r_{B}\right) / 4$ males each of type $A$ and $B$ and $\frac{1}{2}\left[1-\left(r_{A}+r_{B}\right) / 2\right]$ females each of type $A B$ and $B B$ are produced in a colony initiated by an inseminated female of type $A B . B$.

\subsection{Polyandry}

When queens mate with more than one male, they are assumed to mate with males of different genotypes in the proportion that males of these genotypes are represented in the population (at the nest site in the case of sibmating or at the mating aggregate in the case of outbreeding). Equal numbers of sperms of each male are assumed to be stored in the spermatheca which are then used randomly. Thus in a system of 2 alleles, if every female mates twice there will be 9 types of inseminated females, $A A . A . A, A A . A . B$, $A A . B . B, A B . A . A, A B . A . B, A B . B . B, B B . A . A, B B . A . B$, and BB.B.B, where the first two letters refer to the genotype of the female and the last two letters refer to the genotypes of the 2 males she has mated with. Similarly, one can write down the genotypes of inseminated females for any specified number of matings. For different numbers of matings we have investigated the outcome of competition between alternative sex ratio alleles both under queen and worker control.

\subsection{Evolution of kin recognition}

In the previous section the workers were assumed to invest in all brothers and sisters irrespective of their relatedness (i.e., full sisters and half sisters were not distinguished). Here we investigate the case where workers can distinguish genetic relatedness and will invest in their full sisters, but not their half sisters. Multiple mating by the queen does not make any difference in the case of brothers as there can be no half brothers in a haplodiploid system.

We also model kin recognition by a one-locus-two allele system. Now allele $A$ in addition to coding for $r_{A}$ proportion of males also confers ability to recognise kin. $B$ on the other hand codes for $r_{A}$ proportion of males but does not confer ability to recognise kin. Instead of assuming that if the ability to recognise kin is dominant, the sex ratio specified by that allele is necessarily dominant, we have considered all possible combinations of dominance and recessiveness of kin recognition ability and sex ratio. 
We have considered a two-insemination case for simplicity. This implies nine types of inseminated females, $A A . A . A, A A . A . B, \ldots B B . B . B$. The contributions to the next generation from each of these genotypes are calculated in a manner analogous to the queen control case described in the appendix.

The invadability of $A$ and $B$ by each other for various values of $r_{A}, r_{B}$ and $d$ was investigated using procedures similar to those described in the earlier sections.

\subsection{Optimal sex ratio under kin recognition}

Here we assume that the kin recognition gene has gone to fixation and investigate how the competing genotypes specifying alternative sex ratio strategies would fare against each other. We again consider a two-insemination case. As mentioned earlier since there are no half brothers each worker treats all the males in the nest equally. On the other hand, the investment meant for the female reproductives is used only for its full sisters, ignoring the half sisters completely.

For example, in a colony founded by a $A A . A . B$ female, the daughters would be of genotype $A A$ and $A B$, each type being full sisters amongst themselves but half sisters of each other. Hence $A A$ females would invest only in $A A$ females and brothers, while $A B$ females would invest only in $A B$ females and brothers. In a colony founded by a female of the type $A B . A . A$ on the other hand, there would still be two lines of full sisters but both would contain equal numbers of females of the genotype $A A$ and $A B$. In other words full sisters and half sisters are decided by which father the sperm has come from and not by the genotype with reference to the alleles $A$ and $B$ in our model; these alleles are merely sex investment ratio determining alleles. We assume therefore that both $\boldsymbol{A} \boldsymbol{A}$ and $A B$ females would invest in males on the one hand and equally in $A A$ and $A B$ females on the other. In contrast, in a colony founded by females of the type $A B . A . B$ there would again be two lines of full sisters, one consisting of $A A$ and $A B$ females and the other of $B B$ and $A B$ females. While each type of worker will allocate resources between brothers and sisters depending on its genotype, resources meant for the sisters will be subdivided in the following manner. $A A$ workers will invest equally in $A A$ females and $50 \%$ of the $A B$ females who are their full sisters. $B B$ females will similarly invest equally in $B B$ females and the remaining $50 \%$ of $A B$ females who are their full sisters. As for $A B$ females, the first $50 \%$ who are full sisters of $A A$ females will invest equally in $A \boldsymbol{A}$ females and in that $50 \%$ of $A B$ females who are their full sisters. Similarly the other $50 \%$ of $A B$ females who are full sisters of $B B$ females will invest equally in $B B$ females and the remaining $50 \%$ of $A B$ females who are their full sisters. Using this formulation, we have investigated the outcome of competition between alternate sex ratio alleles under worker control when kin recognition is present.

\section{Results}

\subsection{Evolutionarily stable strategies}

What would be the sex investment strategies favoured by Natural Selection? We should look for those strategies which would go to fixation against competition from any other strategy. This can be readily done by a glance at the fate maps which present the results of competition between pairs of genotypes specifying different sex investment ratios. 
We illustrate this with schematic diagrams in figure 1 where values on the abscissa are proportions of male offspring specified by the dominant allele $A$ and those on the ordinate by the recessive allele $B$. The diagonal is an equifitness line where the two alleles are indistinguishable with respect to the sex ratios. As described earlier, when the largest eigenvalue $(\lambda)$ of the transition matrix $G$ (appendix) is greater than 1 , the resident allele can be invaded, whereas if it is less than 1 it cannot be invaded (in all the cases investigated the largest eigenvalue was always real). The set of pairs of $r_{A}$ and $r_{B}$ which correspond to $\lambda=1$ thus forms a boundary between regions of stability and instability. Two such lines, along with the diagonal, divide the coordinate space into three kinds of regions (figure 1) viz A, where the dominant allele $A$ goes to fixation, B, where the recessive allele $B$ goes to fixation and $C$, where the two alleles coexist.

To see which strategy will be favoured by natural selection notice from figure la that when the dominant allele takes a value $\hat{r}_{A}$ it would go to fixation irrespective of the value taken by the other allele. In other words, this value of $\hat{r}_{A}$ represents an evolutionarily stable strategy (Ess) (Maynard Smith 1974; for a lucid introduction to the concept of ESS, see Maynard Smith 1982).

It is possible, however, that no true Ess exists. In figure $1 \mathrm{~b}$ for instance, whatever value one of the alleles takes, there is always a strategy open for the competing allele to invade. Notice that in figure $1 \mathrm{~b}$ if the allele $A$ takes the value $\hat{r}_{A}$ it may be invaded but never eliminated altogether, no matter what value is taken by the allele $B$. We shall henceforth call this an Ess of the coexistence kind. When the two lines separating the regions of stability and instability do not intersect the diagonal at the same point there emerges a fourth kind of region in the co-ordinate space, a region of frequency dependence denoted by $F$ in figure $1 \mathrm{c}$. In this region that allele goes to fixation which has a higher initial frequency. Now if allele $A$ takes the value $\hat{r}_{A}$ it is stable against invasion by any strategy of the competing allele. However $\hat{r}_{A}$ cannot be called a true ESS because there are also some strategies that the competing allele can adopt against invasion by $\hat{r}_{A}$ (within the region of frequency dependence). We shall henceforth refer to this as an ess of the frequency dependence kind. The conditions under which these different kinds of Ess will obtain are described in detail in Gadgil et al $(1980,1983)$.

a

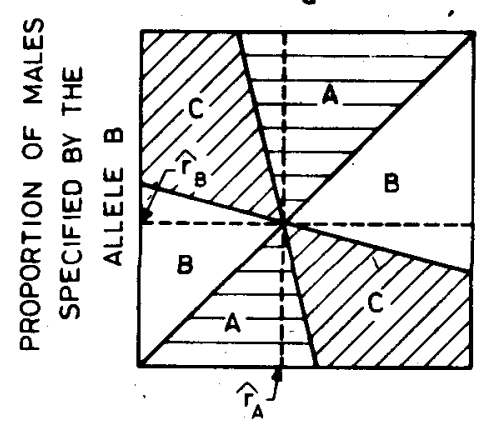

PROPORTION b

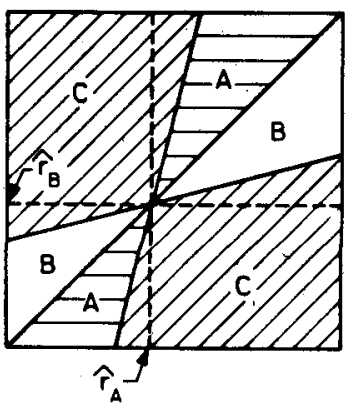

OF MALES SPECIFIED BY

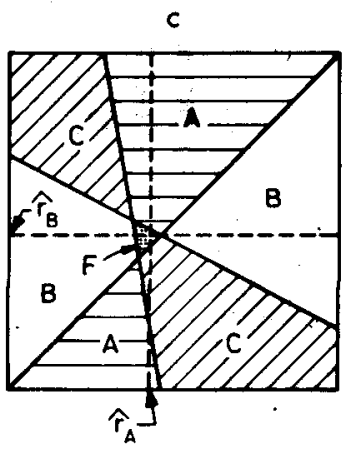

Figure 1. Illustrative fate maps showing schematically (a) true ESS, (b) ESS of the coexistence kind, and (c) ESS of the frequency dependence kind. In the coordinate space of the proportion of males specified by two alleles, $A$ is the area where allele $A$ goes to fixation, $B$, the area where allele $B$ goes to fixation, $C$, where the two alleles coexist, and $F$, the region of frequency dependence (shown as dotted region in c). $r_{A}$ is the ess for allele $A$ and $r_{B}$ the Ess for allele $B$. 


\subsection{Single mating}

3.2a When queens control the ratio of investment: In social insect colonies the queens normally lay all the eggs while the workers (who are often the queens' daughters) are sterile and care for their siblings. Because of haplodiploidy the genetic relationship between a mother and her offspring are different from that of a female and her siblings. This leads to different optimum sex investment ratios for the queens and workers. As we have seen earlier, either the queen or the worker could win in such a conflict. Let us begin by considering the situation where queens (those who have mated with only one male) decide the investment ratios. We have studied the competition between alleles specifying different sex investment ratios under different levels of local mate competition $(d)$. A typical fate map (figure 2) (at $d=0.5$ i.e., where half the offspring disperse before mating while the other half undergo sibmating) shows that the pattern is similar to that depicted in figure $1 \mathrm{~b}$ where no true ess is possible but only an Ess of the coexistence kind. This means that a population need not necessarily consist of a unique strategy but that more than one strategy may coexist leading to the possibility of sex ratio polymorphism.

Variations of this Ess (of the coexistence kind) with levels of LMC (figure 3) show that the results are as expected from the work of Fisher (1930) and Hamilton (1967). Under complete outbreeding $(d=1.0)$ an equal investment in males and females is predicted. As the level of LMC increases the optimum ratio becomes increasingly female biased. The quantitative relationship ${ }^{\dagger}$ between the Ess and $d$ in figure 3 is described by

$$
r=d(1+d) /(3+d)
$$

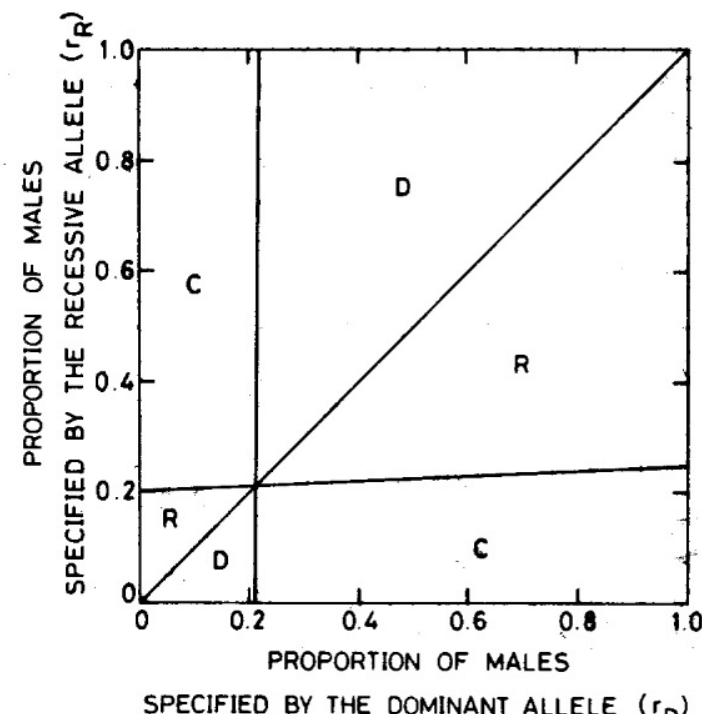

Figure 2. Fate map for competition between a dominant and recessive allele specifying different sex ratios under queen control. The regions denoted by $D, R$ and $C$ correspond to the dominant allele going to fixation, the recessive allele going to fixation and stable coexistence of the two alleles respectively.

\footnotetext{
${ }^{+}$We have not derived this and subsequent equations analytically as the algebra involved is rather tedious. However the equations reproduce the corresponding curves exactly.
} 


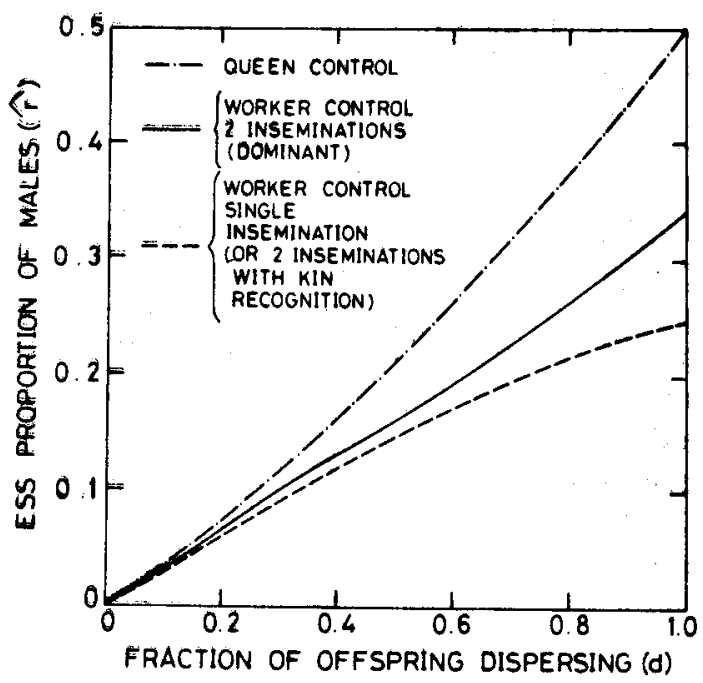

Figure 3. Ess proportion of males $(\hat{r})$ is plotted as a function of the fraction of offspring dispersing $(d)$.

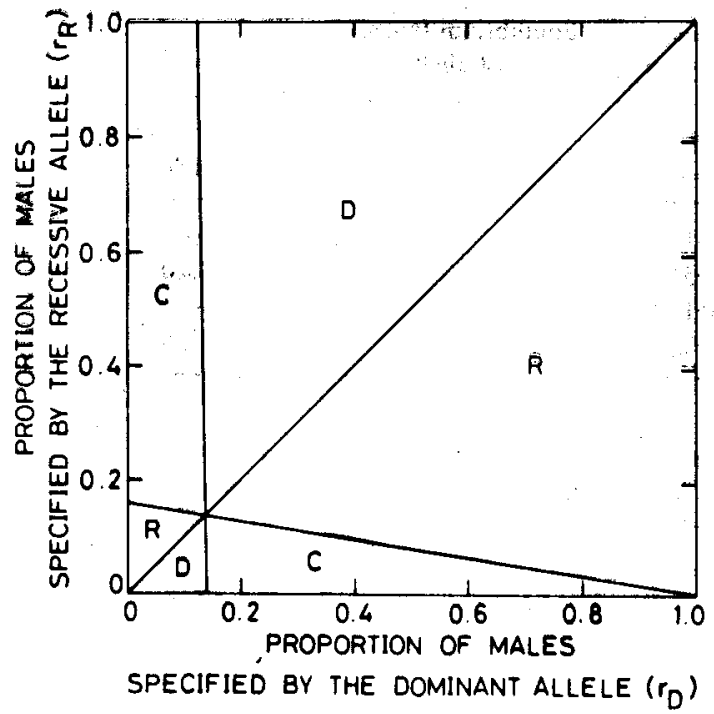

Figure 4. Fate map for competition between dominant and recessive alleles specifying sex ratios under worker control in colonies with single inseminated queens or double insemination with kin recognition. The regions denoted by $D, R$ and $C$ correspond to the dominant allele going to fixation, the recessive allele going to fixation and stable coexistence of the two alleles respectively.

3.2b When workers control the ratio of investment: Now let us consider the situation when workers rather than queens decide the optimum ratio of allocation of resources between the two sexes. Once again in colonies with singly mated queens we have studied competition, under different levels of local mate competition, between alleles specifying different sex allocation ratios. A typical fate map (at $d=0.5$ ) (figure 4 ) shows that the 
pattern is similar to that depicted in figure 1a where a true ess exists. Notice however that the fate map is asymmetrical, with the regions where the dominant allele goes to fixation being smaller than those where the recessive allele does. For instance (figure 4), a recessive allele producing $10 \%$ males always eliminates a dominant allele that produces $50 \%$ males. On the other hand, if the allele specifying $10 \%$ males is dominant and the allele specifying $50 \%$ males is recessive then both alleles coexist at frequencies of 0.734 and 0.256 for the dominant and recessive alleles respectively (table 1 , columns $1-4)$. In other words, a recessive allele goes to fixation while a dominant allele with the same properties fails to do so. Notice that this is different from the well known phenomenon of it being harder to completely eliminate a recessive disadvantageous allele when compared to a dominant disadvantageous allele.

We do not completely understand this rather surprising result but offer the following speculation. Consider how the sex ratio is determined in colonies having more than one genotype. For example in a system of 2 alleles, each colony being initiated by one singly inseminated female, there would be six types of colonies (table 2, row 1). From the genotypes of the queens and that of their mates we can write down the genotypic

Table 1. Genotypic and phenotypic equilibrium sex ratios for dominant and recessive alleles

\begin{tabular}{|c|c|c|c|c|c|}
\hline \multicolumn{2}{|c|}{$\begin{array}{l}\text { Proportion of males } \\
\text { specified by genotypes }\end{array}$} & \multicolumn{2}{|c|}{$\begin{array}{l}\text { Equilibrium frequency } \\
\text { of alleles }\end{array}$} & \multicolumn{2}{|c|}{$\begin{array}{l}\text { Proportion of males obser- } \\
\text { ved at equilibrium }\end{array}$} \\
\hline $\begin{array}{l}\text { Dominant } \\
\text { allele }\end{array}$ & $\begin{array}{c}\text { Recessive } \\
\text { allele }\end{array}$ & $\begin{array}{l}\text { Dominant } \\
\text { allele }\end{array}$ & $\begin{array}{l}\text { Recessive } \\
\text { allele }\end{array}$ & $\begin{array}{l}\text { Dominant } \\
\text { allele }\end{array}$ & $\begin{array}{l}\text { Recessive } \\
\text { allele }\end{array}$ \\
\hline 0.5 & 0.1 & 0.0 & 1.0 & - & 0.1 \\
\hline 0.1 & 0.5 & 0.734 & 0.266 & 0.120 & 0.198 \\
\hline 0.7 & 0.1 & 0.0 & 1.0 & - & 0.1 \\
\hline 0.1 & 0.7 & $0-804$ & 0.196 & 0.118 & 0.206 \\
\hline
\end{tabular}

Table 2. Genotypes of queens, reproductive and worker offspring, and sex ratio of reproductive offspring in different types of nests in a system of one-locus two alleles coding for sex ratio

\begin{tabular}{lccccccc}
$\begin{array}{l}\text { Genotype of inseminated } \\
\text { queen }\end{array}$ & $A A . A^{\dagger}$ & $A A . B$ & $A B . A$ & $A B . B$ & $B B . A$ & $B B . B$ \\
$\begin{array}{l}\text { Genotype of male repro- } \\
\text { ductive offspring }\end{array}$ & $A$ & $A$ & $A$ or $B$ & $A$ or $B$ & $B$ & $B$ \\
$\begin{array}{l}\text { Genotype of female repro- } \\
\text { ductive offspring }\end{array}$ & $A A$ & $A B$ & $A A$ or $A B$ & $A B$ or $B B$ & $A B$ & $B B$ \\
$\begin{array}{l}\text { Genotype of worker off- } \\
\text { spring }\end{array}$ & $A A$ & $A B$ & $A A$ or $A B$ & $A B$ or $B B$ & $A B$ & $B B$ \\
$\begin{array}{l}\text { Sex ratio of reproductives } \\
\text { when } A \text { is dominant }\end{array}$ & $r_{A}$ & $r_{A}$ & $r_{A}$ & $\left(r_{A}+r_{B}\right) / 2$ & $r_{A}$ & $r_{B}$ \\
$\begin{array}{l}\text { Sex ratio of reproductives } \\
\text { when } A \text { is recessive }\end{array}$ & $r_{A}$ & $r_{B}$ & $\left(r_{A}+r_{B}\right) / 2$ & $r_{B}$ & $r_{B}$ & $r_{B}$ \\
\hline
\end{tabular}

+The first two letters refer to the genotype of the queen while the third letter refers to the genotype of the male she has mated with i.e., the sperm she carries in her spermatheca. 
composition of the reproductive brood as well as of the workers in each type of colony (table 2, rows 2-4). Some colonies are pure with respect to $A$ or $B$ while others have a substantial representation of both alleles. Let the allele $A$ be advantageous compared to $B$. When $A$ is dominant, the phenotypic sex ratio corresponds to that advantageous value specified by $A$, both in the colonies with pure $A$ as well as to some extent in the mixed colonies (table 2, row 5). Thus some of this advantage is also conferred on the allele $B$ as a result of which it is never completely eliminated. On the contrary when $A$ is recessive the phenotypic sex ratio corresponds to this value only in the pure $A$ colonies so that none of the advantage is lost to the allele $B$ (table 2, row 6 ). In such a situation $A$ is therefore able to eliminate $B$ altogether. This kind of asymmetry between a dominant and recessive allele seems, therefore, to be because of the fact that the advantageous dominant allele also biases the sex ratio of the bearers of the other allele in the favoured direction, whereas a recessive allele does not do so. If this argument were correct one would predict that when $A$ is dominant and the two alleles coexist, the phenotypic sex ratio of $B$ at equilibrium should be closer to that specified by $A$ (advantageous) rather than by $B$ (disadvantageous). This is indeed what is seen when we look at the phenotypic sex ratios of the two coexisting alleles at equilibrium (table 1, columns 5 and 6).

We saw in the previous section that local mate competition leads to a female biased sex ratio under queen control. When workers control the ratio of investment this optimum sex ratio is even more female biased (figure 3). The quantitative relationship between ESS sex ratio and levels of LMC $(d)$ is described by

$$
r=d /(3+d)
$$

In the absence of LMC $(d=1.0)$ the optimum sex ratio under worker control is $3: 1$ in favour of females as predicted by Trivers and Hare (1976).

\subsection{Polyandry}

When queens mate with males of more than one genotype their daughters would no longer be all full sisters of each other. Since some of the daughters of the queen become reproductives and others workers, the genetic relationship between an average worker and the average reproductive would not be $3 / 4$ as in the case of single mating but lower. The optimum sex ratio from the workers point of view would therefore be different as a consequence of polyandry. The genetic relationship between the queen and her daughters however, is not altered by multiple mating. The optimum sex ratio from the queen's 'point of view' is therefore not altered by polyandry.

As expected, our analysis shows that the results remain unaltered under polyandry when queens control the ratio of investment. On the other hand, there are striking differences in the results under worker control. Considering the case of 2 matings we have studied the competition of alleles specifying different sex ratios, once again under different levels of LMC. The results show that the fate maps (shown for $d=0.5$ in figure 5) are now similar to that shown in figure 1c where a region of frequency dependence emerges. This leads to an ESS of the frequency dependence kind. Besides, the Ess for the dominant allele is different (although only slightly) from that for the recessive allele. The kind of asymmetry between the dominant and recessive alleles seen in the single mating case still persists although considerably reduced. This is probably because 


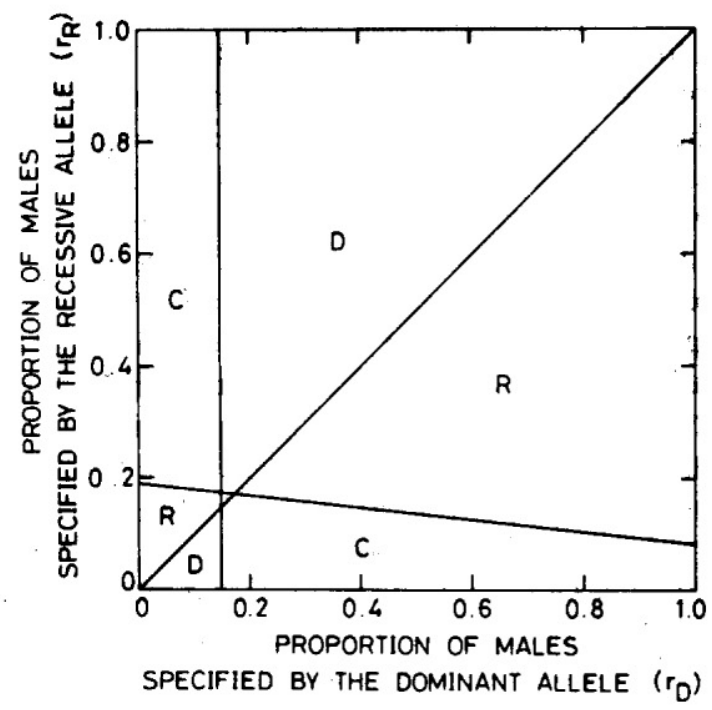

Figure 5. Fate map for competition between a dominant and a recessive allele specifying different sex ratios under worker control in colonies with doubly inseminated queens. The regions denoted by $\mathrm{D}, \mathrm{R}$ and $\mathrm{C}$ correspond to the dominant allele going to fixation, the recessive allele going to fixation and stable coexistence of the two alleles respectively.

with an increase in the number of inseminations, the varieties of colonies with respect to their genotypic composition increases. As a result, even for recessive alleles, its advantage is no longer as strictly confined to itself as before.

When the Ess is plotted as a function of the levels of LMC (figure 3) we see that for any given value of $d$ the optimum sex ratio under worker control with doubly inseminated queens is more female biased than in the case of queen control but more male biased than in the case of worker control with singly inseminated queens. As the number of inseminations that the queen has undergone increases, the optimum sex ratio under worker control becomes increasingly male biased and asymptotically approaches the corresponding value under queen control for that particular value of $d$ (figure 6).

\subsection{Evolution of kin recognition}

As expected, the kin recogniser allele goes to fixation against the non-kin recogniser, almost independently of the sex ratio values specified by these two. This holds whether the kin recogniser is dominant or recessive and whether the kin recogniser codes for a dominant sex ratio trait or the recessive one. In other words, we would expect the kin recogniser allele to go to fixation rapidly in a population once it arises.

In the next subsection, therefore, we describe evolution of optimal sex ratios once kin recognition has been established in the population.

\subsection{Optimum sex ratios in a population with kin-recognition}

The optimum sex ratio under worker control is less female biased when the queen mates more than once because the colony would now contain more than one line of full sisters. 


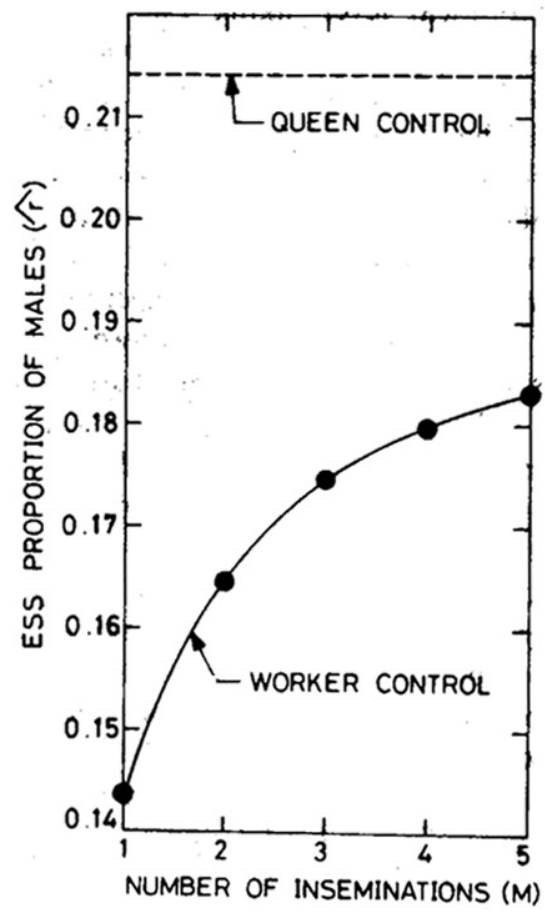

Figure 6. ESs proportion of males $(\hat{r})$ is plotted as a function of number of inseminations $(M)$.

This reduces the average relatedness between a worker and a reproductive female. If by means of kin recognition each worker only invested in her brothers and full sisters one would expect that the optimum sex ratio would be similar to the case where the queen mates only once. We find in fact that under conditions of kin recognition in a colony with doubly mated queens the fate map and the Ess vs $d$ plot are identical to the case where the queens mate only once (figures 3 and 4).

\section{Discussion}

The social insects provide a very attractive model system to study the evolution of sociality in animals. In consequence, considerable theoretical work has gone into this question, and several theories have been proposed primarily to explain the evolution of sterile castes in social insects. Trivers and Hare (1976) used the theory of sex allocation and derived powerful predictions to distinguish between two such theories viz kin selection and parental manipulation and also presented empirical evidence seemingly in overwhelming support of the kin selection theory. Their conclusion unfortunately remains equivocal as a result of their failure to take into consideration two additional factors, LMC and polyandry either in their model or in their data (Alexander and Sherman 1977).

Nonetheless we find their approach of using the concept of sex allocation to derive mutually exclusive predictions, in the frame work of kin selection on the one hand and parental manipulation on the other, very attractive. We have therefore reinvestigated 
this question at the theoretical level taking LMC and polyandry explicitly into consideration. We have calculated the optimum sex allocation ratios from the points of view of the queen (parental manipulation) and workers (kin selection) at different levels of LMC and polyandry. All other things being equal LMC makes the sex ratio increasingly female biased under both situations. Polyandry however has no influence on the sex ratio under queen control but leads to a relatively male biased sex ratio under worker control. In investigating competition between alleles specifying different sex investment ratios we have normally considered the two competing alleles to be a dominantrecessive pair. Considering them to be codominant, however, does not qualitatively alter any of our conclusions although the fate maps, as one might expect, become symmetrical.

A rather unexpected result to emerge from our studies is an asymmetry between the dominant and recessive alleles such that for a particular pair of sex ratio strategies one of them would go to fixation if it is recessive but not if it is dominant. In the latter case, it would coexist with its competitor. We have sought to explain this phenomenon by showing that an advantageous dominant allele does not go to fixation because it also biases the sex ratio of its competitor while a recessive allele's effect on the phenotypic sex ratio is largely confined to itself. We would like to point out that if this explanation were correct it would have wider implications for the evolution of altruistic behaviour; in fact, for any trait that makes its bearer behave altruistically towards a conspecific without regard to the degree of genetic relatedness.

Maynard Smith (1974) has introduced the concept of evolutionarily stable strategy which is defined as a strategy which is unbeatable by any other (schematically illustrated in figure 1a, see also figure 4). In our analysis we found two additional kinds of evolutionarily stable strategies namely that of the coexistence kind (schematically illustrated in figure $1 \mathrm{~b}$, see also figure 2), which guarantees its bearer at least coexistence with any possible competitor, and of the frequency dependence kind (schematically illustrated in figure 1c, see also figure 5), which guarantees its bearer fixation against any competitor provided it is the first to arrive. In our present analysis, these two additional kinds of ess may be taken to be true ess for all practical purposes. In figure 2 showing the ess of the coexistence kind, the frequency of the competitor to the Ess falls off very sharply as we move away from the ess itself. Similarly in figure 5 representing ESS of the frequency dependence kind, the region of frequency dependence is very small: While small in magnitude, these are by no means artefacts and might well turn out to be phenomena of greater significance in other situations.

Taylor and Bulmer (1980) have modelled a scenario consisting of a number of patches, each being colonised by one or more inseminated females, and the offspring mating within the patch before dispersal. Working only within the context of queen control they derive a relationship between the optimum investment ratio $(r)$ and number of females $(n)$ colonising a patch,

$$
r=[(n-1)(2 n-1)] /[n(4 n-1)] .
$$

Here LMC is a function of $n$. In a patch colonised by $n$ females, the proportion of offspring of any female undergoing sibmating would be $1 / n$ which is equivalent to $1-d$ in our model. Equating $d$ to $(n-1) / n,(3)$ is equivalent to our expression for the optimum sex ratio under queen control (1). It must be pointed out that Hamilton (1979) has also arrived at the same expression while modelling dimorphism in fig wasps and that Herre (1985) has derived a more general expression delineating the effects of LMC and inbreeding. 
As expected the ability to recognise kin and invest differentially in full sisters as opposed to half sisters completely counteracts the effects of multiple mating. This result therefore suggests that multiple mating need not necessarily pose any problems for the operation of kin selection. On the other hand, the fact that kin recognition is possible does not automatically provide evidence for kin selection. Even under multiple mating and kin recognition different sex ratios are expected under queen (parental manipulation) and worker (kin selection) control.

Our results emphasize the need to determine the probable levels of LMC and multiple mating, and the possibility of workers differentially investing in full sisters as opposed to half sisters, under field conditions before drawing any conclusions regarding the outcome of worker-queen conflict in social insect colonies. Consider for instance, a 3:1 ratio being taken as evidence of worker control (Trivers and Hare 1976). Notice from our results in figure 3 that a 3:1 ratio is predicted under (i) worker control for complete outbreeding and single mating, (ii) queen control for $d=0.57$, (iii) worker control with doubly mated queens for $d=0.75$, (iv) worker control for complete outbreeding and double mating but with kin recognition. At present, field data on LMC and polyandry in social hymenoptera are rather scanty. Kin recognition has been repeatedly demonstrated (Greenberg 1979; Getz and Smith 1983; Breed 1981; Klahn and Gamboa 1983) but the ability to distinguish between full and half sisters within a single colony has not been demonstrated so far (Gadagkar 1985b). An empirical study designed to simultaneously gather quantitative information on sex investment ratios, LMC, multiple mating and kin recognition is now essential and well within the realm of possibility due to the increasing application of electrophoretic methods (Metcalf 1980). We would like to point out however that factors other than LMC, parental manipulation and kin recognition may also significantly affect the sex ratio. It may therefore be necessary to take these also into account. Significant among such additional factors might be worker oviposition (Benford 1978; Owen and Plowright 1982), orphaned colonies producing male biased sex ratios leading to female biased sex ratios among the remaining queen-right colonies (Owen et al 1980), multi-locus control of sex ratios leading to sex ratio polymorphism (Pamilo 1982), fluctuating sex ratios because of populations being away from equilibrium (Herbers 1979; MacNair 1978), and sex ratio being responsive to proximate factors (Herre 1985; Werren 1980, 1983).

\section{Appendix}

We describe the procedure for obtaining the frequency distribution of the different types of inseminated females at the $(n+1)$ th generation, given the distribution at the $n$th generation. For the two alleles $A$ and $B$ (with $A$ dominant over $B$ ), the proportion of male offspring is $r_{A}$ and $r_{B}$ respectively. A fraction $d$ of the offspring (of both the sexes) disperses from the nest to join a mating pool, while sibmating takes place in the fraction $1-d$.

For the queen control, single insemination case, let the frequencies of the six possible types of inseminated females be denoted by $P_{A A . A}, P_{A A . B}, \ldots, P_{B B . B}$ (where the first two letters of the subscript stand for the genotype of the female and the third for the genotype of the male she has mated with).

From table A1, it can be seen that the total number of females produced would be given by (assuming, without loss of generality, the brood size to be unity),

$$
N_{f}=\left(P_{A A . A}+P_{A A . B}+P_{A B . A}+P_{A B . B}\right)\left(1-r_{A}\right)+\left(P_{B B . A}+P_{B B . B}\right) r_{B}
$$


Table A1. Contributions from the six types of females under queen control, single insemination

\begin{tabular}{|c|c|c|c|c|c|c|c|c|}
\hline & \multirow{2}{*}{$\begin{array}{c}\text { Contri- } \\
\text { bution to }\end{array}$} & \multicolumn{5}{|c|}{ Contribution from } & \multicolumn{2}{|c|}{$\therefore$} \\
\hline & & $A A \cdot A$ & $A A . B$ & $A B \cdot A$ & AB.B & $B B . A$ & . & $B B . B$ \\
\hline \multirow{5}{*}{$\begin{array}{l}\text { Mating } \\
\text { aggregate }\end{array}$} & $A$ & $d r_{A}$ & $d r_{A}$ & $\frac{1}{2} d \cdot r_{A}$ & $\frac{1}{2} d \cdot r_{A}$ & - & & - \\
\hline & $B$ & - & - & $\frac{1}{2} d \cdot r_{A}$ & $\frac{1}{2} d \cdot r_{A}$ & $d r_{B}$ & & $d r_{B}$ \\
\hline & $A A$ & $d\left(1-r_{A}\right)$ & - & $\frac{1}{2} d\left(1-r_{A}\right)$ & - & - & & - \\
\hline & $A B$ & - & $d\left(1-r_{A}\right)$ & $\frac{1}{2} d\left(1-r_{A}\right)$ & $\frac{1}{2} d\left(1-r_{A}\right)$ & $d\left(1-r_{B}\right)$ & & - \\
\hline & $B B$ & $\therefore-$ & - & - & $\frac{1}{2} d\left(1-r_{A}\right)$ & - & & $d\left(1-r_{B}\right)$ \\
\hline \multirow{6}{*}{$\begin{array}{l}\text { Inseminated } \\
\text { females }\end{array}$} & $A A \cdot A$ & $(1-d)\left(1-r_{A}\right)$ & - & $\frac{1}{4}(1-d)\left(1-r_{A}\right)$ & $\therefore-$ & - & & - \\
\hline & $A A . B$ & - & - & $\frac{1}{4}(1-d)\left(1-r_{A}\right)$ & - & - & & $\div$ \\
\hline & $A B . A$ & - & $(1-d)\left(1-r_{A}\right)$ & $\frac{1}{d}(1-d)\left(1-r_{A}\right)$ & $\frac{1}{4}(1-d)\left(1-r_{A}\right)$ & - & & - \\
\hline & $A B . B$ & - & - & $\frac{1}{4}(1-d)\left(1-r_{A}\right)$ & $\frac{1}{4}(1-d)\left(1-r_{A}\right)$ & $(1-d)\left(1-r_{B}\right)$ & & - \\
\hline & BB.A & 8. & - & $\leftarrow$ & $\frac{1}{4}(1-d)\left(1-r_{A}\right)$ & - & & - \\
\hline & $B B . B$ & - & - & - & $\frac{1}{4}(1-d)\left(1-r_{A}\right)$ & - & & $(1-d)\left(1-r_{B}\right)$ \\
\hline
\end{tabular}


The number of $A A$ females in the mating pool is given by

$$
M_{A A}=d . P_{A A . A}\left(1-r_{A}\right)+(d / 2)\left(1-r_{A}\right) P_{A B . A} .
$$

The number of $A$ males in the mating pool is

$$
M_{A}=d . r_{A}\left(P_{A A . A}+P_{A A . B}+\frac{1}{2} P_{A B . A}+\frac{1}{2} P_{A B . B}\right) \text {. }
$$

Hence, the number of $A A$ females which will be inseminated by $A$ males is given by

$$
O_{A A . A}=M_{A A} \cdot M_{A} / d N_{m},
$$

since $d N_{m}$ is the total number of males in the pool. It is also seen from table A1 that the number of inseminated females of the type $A A . A$ resulting from sibmating is given by

$$
S_{A A . A}=(1-d)\left(1-r_{A}\right)\left(P_{A A . A}+P_{A B . A} / 4\right) \text {. }
$$

Hence, the frequency of $A A . A$ in the next generation is given by

$$
P_{A A . A}(n+1)=\left(S_{A A . A}+O_{A A . A}\right) / N_{f} \text {. }
$$

In a similar manner, one can compute the frequencies in the next generation for the other types of females. Since all the frequencies add up to one, one needs only five such equations. By suitable algebraic manipulations, in fact, one can write

$$
\begin{aligned}
& {\left[P_{A A . A}(n+1), P_{A A . B}(n+1), \ldots, P_{B B . A}(n+1)\right]^{T}} \\
& =G .\left[P_{A A . A}(n), \ldots P_{B B . A}(n)\right]^{T},
\end{aligned}
$$

where $G$ is a $5 \times 5$ matrix, whose elements are functions of $d, r_{A}, r_{B}$ and $P_{A A A}, \ldots$, $P_{B B . A}$.

If one considers a pure population of $A$ invaded by a small proportion of $B$, the frequencies $P_{A A . B}, \ldots, P_{B B . A}$ would be very small. Denoting the frequency of $P_{A A . A}$ by $1-\varepsilon_{A A . A}$, one can rewrite $(A 1)$ in the form

$$
\begin{aligned}
& {\left[-\varepsilon_{A A . A}(n+1), P_{A A . B}(n+1), \ldots, P_{B B . A}(n+1)\right]^{T}} \\
& =G^{\prime}\left[-\varepsilon_{A A . A}(n), \ldots, P_{B B . A}(n+1)\right]^{T} .
\end{aligned}
$$

Since the terms involving products and higher powers of $\varepsilon_{A A . A}, P_{A A . B}$ etc. can be neglected, the elements of the matrix $G^{\prime}$ become functions only of $r_{A}, r_{B}$ and $d$, i.e., the nonlinear system (A1) is replaced by a linear system (A2).

The eigenvalues of $G^{\prime}$ are now computed and if the real part of the largest eigenvalue is greater than unity, it implies that the allele $B$ can invade a population of pure $A$, while if it is less than unity, the allele $B$ is unable to invade $A$. Stability analysis for other situations like worker control, multiple mating and kin recognition can be carried out in an analogous manner.

\section{Acknowledgements}

It is a pleasure to thank Drs Madhav Gadgil, V Nanjundiah, Eric Charnov and especially Pekka Pamilo for reviewing the manuscript and Drs W D Hamilton and Sulochana Gadgil for many helpful discussions. This work was supported by a grant from the DST, New Delhi. 


\section{References}

Alexander R D 1974 Ann. Rev. Ecol. Syst. 5: 325-383

Alexander R D and Sherman P W 1977 Science 196: 494-500

Benford F A $1978 \mathrm{~J}$. Theor. Biol. 72: 701-727

Breed M D 1981 Proc. Natl. Acad. Sci. U.S.A. 78: 2635-2637

Bulmer M G 1981 J. Theor. Biol. 93: 239-251

Bulmer M G 1983 Am. Nat. 121: 899-902

Charnov E L 1978a Am. Nat. 112: 317-326

Charnov E L 1978b J. Theor. Biol. 75: 451-465

Charnov E L 1982 The theory of sex allocation (Princeton: University Press)

Craig R 1979 Evolution 33: 319-334

Craig R 1980a Am. Nat. 116: 331-323

Craig R 1980b J. Theor. Biol. 87: 55-70

Crozier R H 1980 in Dahlem konferenzen 1980 (ed.) H Markl (Weinheim Verlag Chemie GmbH) pp. 129-146

Crozier R H and Brückner D 1981 Am. Nat. 117: 561-563

Fisher R A 1930 The genetical theory of natural selection (Oxford: Clarendon Press)

Gadagkar R 1985a Proc. Indian Acad. Sci (Anim. Sci) 94: 309-324

Gadagkar R 1985b Proc. Indian Acad. Sci. (Anim. Sci.) (submitted)

Gadgil M, Joshi N V and Gadgil S 1983 J.Theor. Biol. 104: 21-42

Gadgil S, Nanjundiah V and Gadgil M 1980 J. Theor. Biol. 84: 737-759

Getz W M and Smith K B 1983 Nature 302: 147-149

Greenberg L 1979 Science 206: 1095-1097

Hamilton W D 1964a J. Theor. Biol. 7: 1-16

Hamilton W D 1964b J. Theor. Biol. 7: 17-52

Hamilton W D 1967 Science 156: 477-488

Hamilton W D 1979 in Sexual selection and reproductive competition in insects (eds.) M S Blum and N A Blum (New York: Academic Press) pp. 167-220

Herbers J M 1979 Am. Nat. 114: 818-834

Herre E A 1985 Science 228: 896-898

Klahn J E and Gamboa G J 1983 Science 221: 482-484

MacNair M R 1978 J. Theor. Biol. 70: 449-459

Maynard Smith J 1974 J. Theor. Biol. 47: 209-221

Maynard Smith J 1982 Evolution and the theory of games (Cambridge: University Press)

Metcalf R A 1980 Am. Nat. 116: 642-654

Orlove M J 1975 J. Theor. Biol. 49: 289-310

Oster G, Eshel I and Cohen D 1977 Theor. Popul. Biol. 12: 49-85

Owen R E 1983 Oecologia (Berlin) 59: 402-404

Owen R E and Plowright R C 1982 Behav. Ecol. Sociobiol. 11: 91-99

Owen R E. Rodd F H and Plowright R L 1980 Behav. Ecol. Sociobiol. 7: 287-291

Page R E and Metcalf R A 1982 Am. Nat. 119: 263-281

Pamilo P 1982 Am. Nat. 119: 638-656

Starr C K 1984 in Sperm competition and the evolution of animal mating systems (ed.) R L Smith (New York: Academic Press) pp. 427-464

Taylor P D and Bulmer M G 1980 J. Theor. Biol. 86: 409-419

Trivers R L and Hare H 1976 Science 191: 249-263

Uyenoyama $M$ and Bengtsson B O 1981 Theor. Popul. Biol. 20. 57-79

Werren J H 1980 Science 208: 1157-1159

Werren J H 1983 Evolution 37: 116-124

Wilson E O 1971 The insect societies (Cambridge, Mass: Harvard University Press) 